\title{
ON SEQUENCES OF CONTRACTIVE MAPPINGS AND THEIR FIXED POINTS
}

\author{
M. IMDAD \\ Aligarh Muslim University \\ Department of Mathematics \\ Aligarh-202001, India

\section{M.S. KHAN} \\ King Abdul Aziz University \\ Department of Mathematics \\ Faculty of Science \\ P.O. BOX 9028 \\ Jeddah, Saudi Arabia

\section{S. SESSA} \\ Universita di Napoli \\ Facoltà di Architettura \\ Istituto di Matematica \\ Via Monteoliveto, 3 \\ 80134 Napoli, Italy \\ (Received October 12, 1984 and in revised form February 9, 1987)
}

ABSTRACT. By using a condition of Reich, we establish two fixed point theorems concerning sequences of contractive mappings and their fixed points. A suitable example is also given.

KEY WORDS AND PHRASES: Complete metric space, Fixed point, Sequence of mappings. 1980 AMS SUBJECT CLASSIFICATION CODE. 47H10, 54H25.

1. INTRODUCTIONS.

Throughout this paper, $(\mathrm{X}, \mathrm{d})$ denotes a complete metric space and $\mathrm{T}$ stands for a mapping of $X$ into itself. It is well known that each of the following conditions ensure the existence and uniqueness of a fixed point of $\mathrm{T}$ :

(A) (Banach). There exists a number $k, 0 \leqq k<1$, such that for each $x, y$ in $x$,

$$
d(T x, T y) \leqq k \cdot d(x, y)
$$

(B) (Rakotch [1]). There exists a monotonically decreasing function $\mathrm{g}$ : $(0, \infty) \rightarrow[0,1)$ such that for each $x, y$ in $x, x \neq y$,

$$
d(T x, T y) \leqq g(d(x, y) d(x, y) .
$$

(C) (Reich [2]). There exist nonnegative numbers $a, b$ such that for each $x, y$ in $x, x \neq y$,

$$
d(T x, T y) \leqq a \cdot d(x, y)+b \cdot[d(x, T x)+d(y, T y]
$$


(D) (Reich [3]). There exist monotonically decreasing functions $a, b:(0, \infty) \rightarrow[0,1)$ such that for each $x, y$ in $x, x \neq y$,

$$
d(T x, T y) \leqq a(d(x, y)) \cdot d(x, y)+b(d(x, y)) \cdot[d(x, T x)+d(y, T y)],
$$

where for any $t>0$,

$$
a(t)+2 b(t)<1
$$

(E) (Reich [4]). There exist functions $a, b:(0, \infty) \rightarrow[0,1)$ such that (1.1) holds for each $x, y$ in $x, x \neq y$, satisfying $(1.2)$ and

$$
\limsup _{r \rightarrow t^{+}}[a(r)+2 b(r)]<1 \text {. }
$$

(F) (Hardy and Rogers [5]). There exist monotonically decreasing functions a,b,c: $(0, \infty) \rightarrow[0,1)$ such that for each $x, y$ in $x, x \neq y$,

$$
\begin{array}{r}
d(T x, T y) \leqq a(d(x, y) \cdot d(x, y)+b(d(x, y)) \cdot[d(x, T x)+d(y, T y)] \\
+c(d(x, y)) \cdot[d(x, T y)+d(y, T x)],
\end{array}
$$

where for any $t>0$,

$$
a(t)+2 b(t)+2 c(t)<1
$$

It is not hard to show that, adopting the same proof of [4], that $T$ has a unique fixed point if

(G) There exist functions $a, b, c:(0, \infty) \rightarrow[0,1)$ such that (1.3) holds for each $x, y$ in $x, x \neq y$, satisfying $(1.4)$ and

$$
\underset{r \rightarrow t^{+}}{\operatorname{limpsup}}[a(r)+2 b(r)+2 c(r)]<1 \text {. }
$$

Evidently (A) implies (B) and (C), (B) and (C) imply (D), (D) imply (E) and (F), (E) and (F) imply (G). Suitable examples can be found in Rhoades [6] to illustrate some of the above implications. In the sequel, $N$ stands for the set of natural numbers.

The following result was established in [5] and [6].

THEOREM 1. Let $T_{n}$, $n \in N$, be mappings of $(x, d)$ into itself satisfying condition (F) with the same functions $a, b, c$ and with fixed points $z_{n}$. Suppose that a mapping $T$ of $X$ into itself can be defined pointwise by $T(x)=\lim _{n \rightarrow \infty} T T_{n}(x)$ for any $x$ in $X$. Then $T$ has a unique fixed point $z$ and $z=\lim _{n \rightarrow \infty} z_{N}$

Theorem 1 generalizes an analogous result of Bonsal1 [7], Theorem 6 of [2] and Theorem 4 of [3] established for mappings $T_{n}$ satisfying conditions (A), (C) and (D), respectively. Results due to Chatterjea [8] and Singh [9], Kannan type mappings, are also included in Theorem 1 .

The proof of Theorem 1 consists essentially in the fact that the sequence $\left\{z_{n}\right\}$ is regular, i.e. it possesses a limit $z$ (say). It appears that a result corresponding to Theorem 1 for mappings satisfying condition $(G)$ does not exist in print in the 
literature of fixed point theory. In this note, we wish to study this problem. Other results and open problems related to the stability of fixed point of mappings can be found in Rhoades [6], Nadler [10], Rus [11] and Singh [12].

\section{RESULTS.}

We first state the following result more general than Theorem 1 .

THEOREM 2. Let $T_{n}$, $n \in N$, be mappings of $(X, d)$ into itself satisfying condition (G) with the same functions $a, b, c$ and with fixed points $z_{n}$. Suppose that a mapping $T$ of $X$ into itself can be defined pointwise by $T(x)=\lim _{n \rightarrow \infty} T_{n}(x)$ for any $x$ in $X$ and the sequence $\left\{z_{n}\right\}$ is regular. Then $T$ has a unique fixed point $z$ and $z=1$ im $n \rightarrow \infty z_{n}$.

PROOF. Since the metric $d: X \times X \rightarrow[0,+\infty)$ is continuous, the limit mapping $T$ satisfies the inequality (1.3). By condition (G), $T$ has a unique fixed point $z$. We claim that

$$
\ell=\inf _{n \in N} d\left(z_{n}, z\right)=0
$$

otherwise assume $\ell>0$. By observing that $d\left(z_{n}, z\right) \geq \ell>0$ and hence $z_{n} \neq z$ for any $n \in N$, we deduce using (1.3) and the triangular property of the metric $d$,

$$
\begin{aligned}
d\left(z_{n}, z\right) & =d\left(T_{n} z_{n}, T z\right) \leqq d\left(T_{n} z_{n}, T_{n} z\right)+d\left(T_{n} z, T z\right) \\
& \leqq a \cdot d\left(z_{n}, z\right)+b \cdot d\left(z, T_{n} z\right) \\
& +c \cdot\left[d\left(z_{n}, z\right)+d\left(z, T_{n} z\right)+d\left(z, T_{n} z_{n}\right)\right]+d\left(T_{n} z, T z\right) \\
& =(a+2 c) \cdot d\left(z_{n}, z\right)+(1+b+c) \cdot d\left(T_{n} z, z\right)
\end{aligned}
$$

for any $n \in N$, where $a=a\left(d\left(z_{n}, z\right)\right.$ and similarly for $b$ and $c$.

Thus

$$
d\left(z_{n}, z\right) \leqq \frac{2 d\left(T_{n} z, z\right)}{1-(a+2 c)}
$$

for any $n \in N$. If we denote with $\left\{z_{k(n)}\right\}$ a subsequence of $\left\{z_{n}\right\}$ such that

$$
\ell \leq \mathrm{d}\left(\mathrm{z}_{\mathrm{k}(\mathrm{n})}, \mathrm{z}\right)<\ell+1 / \mathrm{n}
$$

we obtain that

$$
\lim _{n \rightarrow \infty} d\left(z_{k}(n), z\right)=\ell>0
$$

Following Reich [4], we oserve that the assumptions about the functions $a, b, c$ of condition (G) imply the existence of two functions $h, k:(0, \infty) \rightarrow(0, \infty)$ for which, given $t>0$, there exists an $h(t)>0$ such that $0 \leqq r-t<h(t)$ implies

$$
a(r)+2 b(r)+2 c(r) \leqq k(t)<1 .
$$

By (2.2), let peN such that

$$
0 \leqq d\left(z_{k}(n), z\right)-\ell<h(\ell)
$$


for any $n \geq p$. From (2.3), it follows that

$$
a\left(d\left(z_{k(n)}, z\right)\right)+2 c\left(d\left(z_{k}(n), z\right)\right) \leqq k(l)<1
$$

for any $n \geq p$. On the other hand, since the sequence $\left\{T_{k(n)}(z)\right\}$ converges to $z=T(z)$, we can find an integer qEN such that

$$
\mathrm{d}\left(\mathrm{Tz}, \mathrm{T}_{\mathrm{k}(\mathrm{n})}(\mathrm{z})\right)<\ell(1-\mathrm{k}(\ell)) / 2
$$

for any $n \geq q$. By (2.1), then we have for any $n \geq \max \{p, q\}$,

$$
\ell \leqq d\left(z_{k(n)}, z\right) \leqq \frac{2 d\left(T z, T_{k(n)}(z)\right)}{1-\left[a\left(d\left(z_{k(n)}, z\right)\right)+2 c\left(d\left(z_{k(n)}, z\right)\right)\right]}<\ell,
$$

a contradiction. Thus $\ell=0$ and therefore the sequence $\left\{z_{k(n)}\right\}$ converges to $z$. Since $\left\{z_{n}\right\}$ is regular, it has limit $z$ and this concludes the proof.

THEOREM 3. Let $T_{n}$ be mappings of $(X, d)$ into itself with at least one fixed point $z_{n}$. If $\left\{T_{n}\right\}$ converges uniformly to a mapping $T$ of $X$ into itself satisfying condition (G) and if the sequence $\left\{z_{n}\right\}$ is regular, then $z=\lim _{n \rightarrow \infty} z_{n}$, where $z$ is the unique fixed point of $T$.

PROOF. We have that

$$
\begin{aligned}
d\left(z_{n}, z\right) & =d\left(z_{n}, T z\right) \leqq d\left(z_{n}, T z_{n}\right)+d\left(T z_{n}, T z\right) \\
& \leqq d\left(z_{n}, T z_{n}\right)+a \cdot d\left(z_{n}, z\right)+b \cdot d\left(z_{n}, T z_{n}\right) \\
& +c \cdot\left[d\left(z_{n}, z\right)+d\left(z_{,} z_{n}\right)+d\left(z_{n}, T z_{n}\right)\right] \\
& =(a+2 c) \cdot d\left(z_{n}, z\right)+(1+b+c) \cdot d\left(z_{n}, T z_{n}\right) .
\end{aligned}
$$

for any $n \varepsilon N$, where $a=a\left(d\left(z_{n}, z\right)\right)$ and similarly for $b$ and $c$. Thus

$$
d\left(z_{n}, z\right) \leqq \frac{2 d\left(z_{n}, T z_{n}\right)}{1-(a+2 c)}
$$

for any neN and proceeding as in the proof of Theorem 2, we get the thesis.

REMARK 1. It is evident that there certainly exists a subsequence of $\left\{z_{n}\right\}$ converging to $z$, even if $\left\{z_{n}\right\}$ is not regular. It is not yet known if the regularity of $\left\{z_{n}\right\}$ is a necessary condition in Theorems 2 and 3 .

REMARK 2. Theorem 3 generalizes Theorem 5 of Ray [13], which in turn extends Theorem 9 of Reich [3].

REMARK 3. Following Ray [13] and Fraser and Nadler [14], one can establish a result analogous to Theorem 10 of Reich [3] by using condition (G). 


\section{AN EXAMPLE.}

In order to illustrate the degree of generality of Theorem 2 over Theorem 1, we furnish an example which shows that there exist mappings $T_{n}$ of $X$ into itself satisfying condition (G) but no condition $(F)$.

EXAMPLE. Let $X=[0,1]$ be equipped with metric $d$ defined as follows,

$$
d(x, y)= \begin{cases}|x-y| & \text { if } x, y \in[0,1] \\ x+y & \text { if one of } x, y \in N-\{1\}\end{cases}
$$

Then $(X, d)$ is a complete metric space because it is isometric to a closed subspace of the space of absolutely summable sequences. For further details, see Boyd and Wong [15].

Now we define $T_{n}: X \rightarrow X, n \in N$, by setting $T_{1}(x)=T_{2}(x)=0$ for any $x$ in $X$ and for $n \geq 3$,

$$
T_{n}(x)= \begin{cases}x-(n-1) x^{2} /(2 n-3) & \text { if } x \in[0,1] \\ x-1 & \text { if } x \in N-\{1\}\end{cases}
$$

Further, $T_{n}$ does not satisfy condition ( $F$ ) for $n \geq 3$, otherwise we should have for $y=0$ and $\mathrm{x}=\mathrm{t} \quad \varepsilon(0,1]$,

$$
\begin{aligned}
t-\frac{(n-1) t^{2}}{2 n-3} & \leq a(t) \cdot t+b(t) \cdot \frac{(n-1) t^{2}}{2 n-3}+c(t) \cdot\left[t-\frac{(n-1) t^{2}}{2 n-3}+t\right] \\
& \leq[a(t)+2 c(t)] \cdot t+[b(t)-c(t)] \cdot \frac{(n-1) t^{2}}{2 n-3} \\
& <[1-2 b(t)] \cdot t+b(t) \cdot \frac{(n-1) t^{2}}{2 n-3} .
\end{aligned}
$$

Since $b(t)<1 / 2$ for any $t>0$, we obtain

$$
1-\frac{(n-1) t}{2 n-3}<1-2 b(t)+\frac{1}{2} \cdot \frac{(n-1) t}{2 n-3}
$$

for any $t \in(0,1]$. This implies, for $n \geq 3$, that

$$
1-t \leq 1-\frac{3}{2} \frac{(n-1) \cdot t}{2 n-3}<1-2 b(t),
$$

i.e. $b(t) \leqq 2 b(t)<t$ for any $t \varepsilon(0,1]$. Then, since $b$ is monotonically decreasing in $(0, \infty)$, we should have $b(t)=0$ for any $t>0$. 
Therefore, for each $x, y$ in $x, x \neq y$, and $n \geq 3$, the condition ( $F$ ) reduces to $d\left(T_{n} x, T_{n} y\right) \leqq a(d(x, y)) \cdot d(x, y)+c(d(x, y)) \cdot\left[d\left(x, T_{n} y\right)+d\left(y, T_{n} x\right)\right]$

Now for $x=0$ and $y=q \varepsilon N-\{1\}$, we deduce that

$$
q-1 \leqq a(q) \cdot q+c(q) \cdot[q-1+q]<a(q) \cdot q+2 c(q) \cdot q \cdot
$$

This implies, since a and c are monotonically decreasing functions, that

$$
\frac{\mathrm{q}-1}{\mathrm{q}}<\mathrm{a}(1)+2 \mathrm{c}(1) .
$$

As $q \rightarrow+\infty$, we obtain $1 \leqq a(1)+2 c(1)<1$, a contradiction which shows that the condition (F) is not satisfied by $T_{n}$ for $n \geqq 3$.

On the other hand, for any $n \in N$ the condition $(G)$ holds if we choose $b(t)=c(t)$ $=0$ for any $t>0$ and $a(t)=1-t / 2$ if $0<t \leqq 1, a(t)=1-1 / t$ if $t>1$. The condition (G) is obviously satisfied by $T_{1}$ and $T_{2}$. For $n \geq 3$ and $x, y$ in $[0,1], x \neq y$, we get

$$
\begin{aligned}
d\left(T_{n} x, T_{n} y\right) & =|x-y| \cdot\left[1-\frac{n-1}{2 n-3} \cdot(x+y)\right] \\
& <|x-y| \cdot\left[1-\frac{1}{2}(x+y)\right] \leqq|x-y| \cdot\left[1-\frac{1}{2}|x-y|\right] \\
& =c(d(x, y)) \cdot d(x, y) .
\end{aligned}
$$

Furthermore, if one of $x, y$ lies in $N-\{1\}$ with $x \neq y$ and $n \geq 3$, then we have

$$
\begin{aligned}
d\left(T_{n} x, T_{n} y\right) & =T_{n} x+T_{n} y \leq x+y-1 \\
& =(x+y) \cdot[1-1 /(x+y)] \\
& +c(d(x, y)) \cdot d(x, y)
\end{aligned}
$$

We now define $T(x)=x-x^{2} / 2$ if $0 \leqq x \leqq 1, T(x)=x-1$ if $x$ is in $N-\{1\}$. Of course, $z_{n}=z=0$ are the unique fixed points of $T$ and $T_{n}$, respectively and we have

$$
\lim _{n \rightarrow \infty} T(x)=T(x)
$$

for any $x$ in $X$. Thus the conclusion of Theorem 2 holds good since the sequence $\left\{z_{n}\right\}$ converges to $z$.

The idea of this example appears in [15].

ACKNOWLEDGEMENT. The authors thank an anonymous referee for some useful suggestions. 


\section{REFERENCES}

1. RAKOTCH, E. A Note on Contractive Mappings, Proc. Amer. Math. Soc. 13 (1962), 459-465.

2. REICH, S. Some Remarks Concerning Contraction Mappings, Canad. Math. Bu11., 14 (1971), 121-124.

3. REICH, S. Kannan's Fixed Point Theorem, Bol1. Un. Mat. Ital., 4 (4) (1971), 1-11.

4. REICH, S. Fixed Points of Contractive Functions, Boll. Un. Mat. Ital., $\underline{5}$ (4) (1972), 26-42.

5. HARDY, G.E. and ROGERS, T.D. A Generalization of a fixed point theorem of Reich, Canad. Math. Bul1., 16 (2) (1973), 201-206.

6. RHOADES, B.E. A Comparison of Various Definitions of Contractive Mappings, Trans. Amer. Math. Soc., 226 (1977), 257-290.

7. BONSALL, F.F. Lectures on Some Fixed Point Theorems of Functional Analysis, Tata Institute of Fundamental Research, Bombay, 1962.

8. CHATTERJEA, S.K. Fixed Point Theorems, Comptes Rend. Acad. Bulgare Sci., 25 (1972), 727-730.

9. SINGH, S.P. Some Results on Fixed Point Theorems, Riv. Mat. Univ. Parma, 17(2) (1969), 61-64.

10. NADLER Jr., S.B. Sequences of Contractions and Fixed Points, Pacific. J. Math., 27 $(1968), 579-585$.

11. RUS, I.A. Results and Problems in the Metrical Fixed Point Theory, Math. Rev. Ana1. Numer. Theor. Approximation, 21(44) 2 (1979), 189-194.

12. SINGH, S.P. On Sequence of Contraction Mappings, Riv. Mat. Univ. Parma, 11 (2) (1970), 227-231.

13. RAY, B.K. On Ciric's Fixed Point Theorem, Fund. Math., 94 (1977), 221-229.

14. FRASER Jr., R.B. and NADLER, Jr., S.B. Sequences of Contractive Maps and Fixed Points, Pacific J. Math., 51 (1969), 659-667.

15. BOYD, D.W. and WONG, J.S.W. On Nonlinear Contractions, Proc. Amer. Math. Soc., $\underline{20}(1969), 458-464$. 


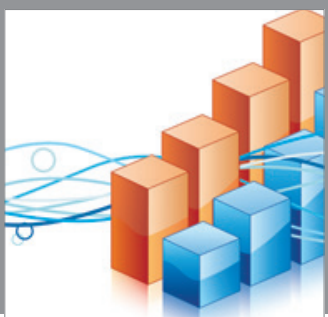

Advances in

Operations Research

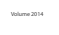

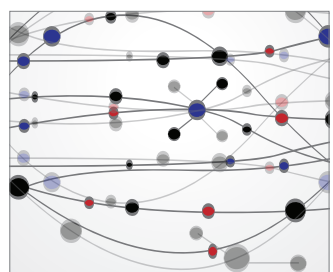

\section{The Scientific} World Journal
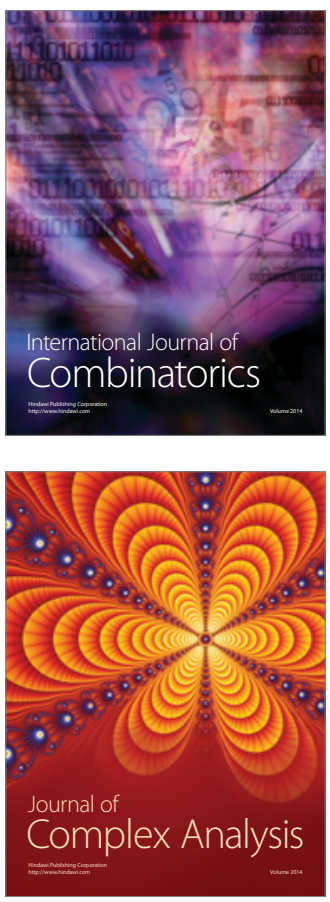

International Journal of

Mathematics and

Mathematical

Sciences
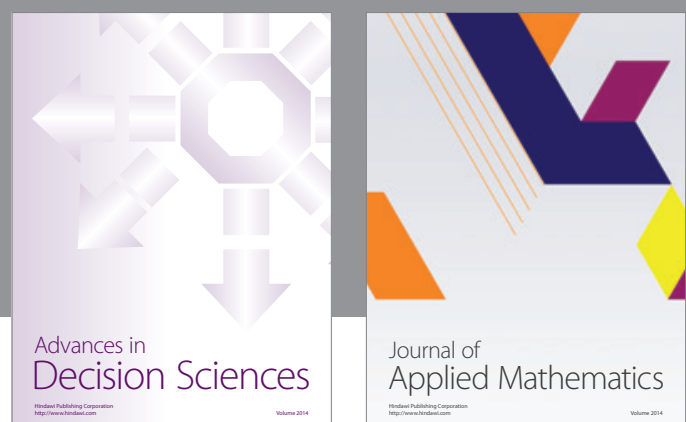

Journal of

Applied Mathematics
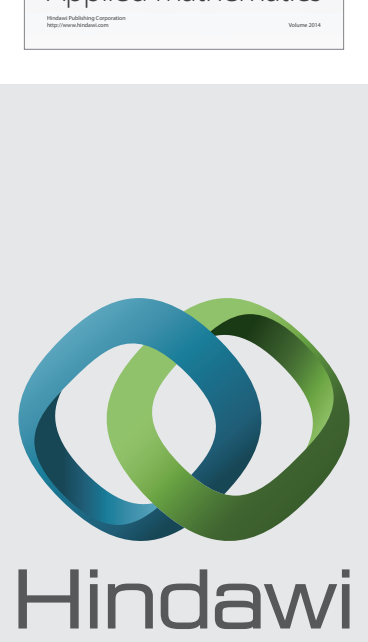

Submit your manuscripts at http://www.hindawi.com
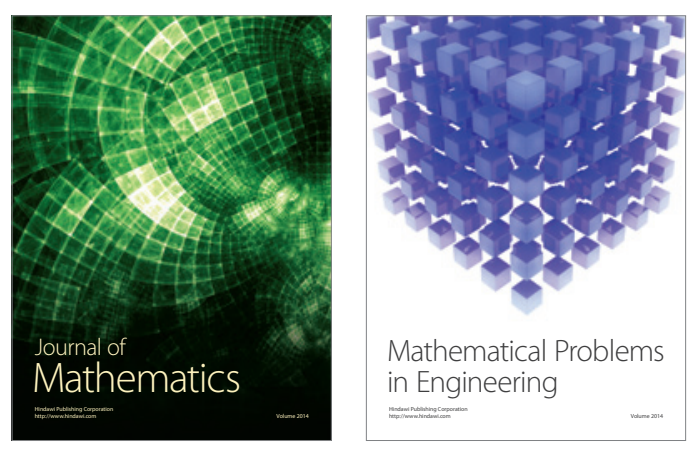

Mathematical Problems in Engineering
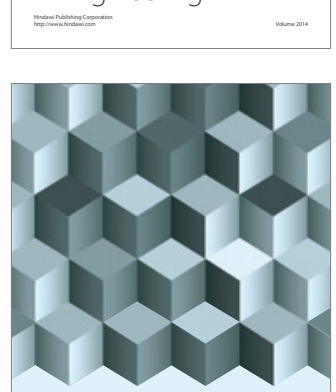

Journal of

Function Spaces
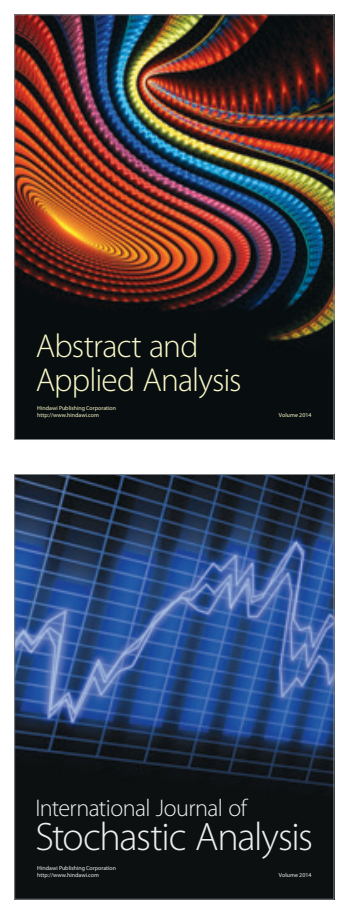

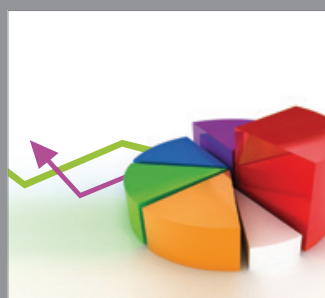

ournal of

Probability and Statistics

Promensencen
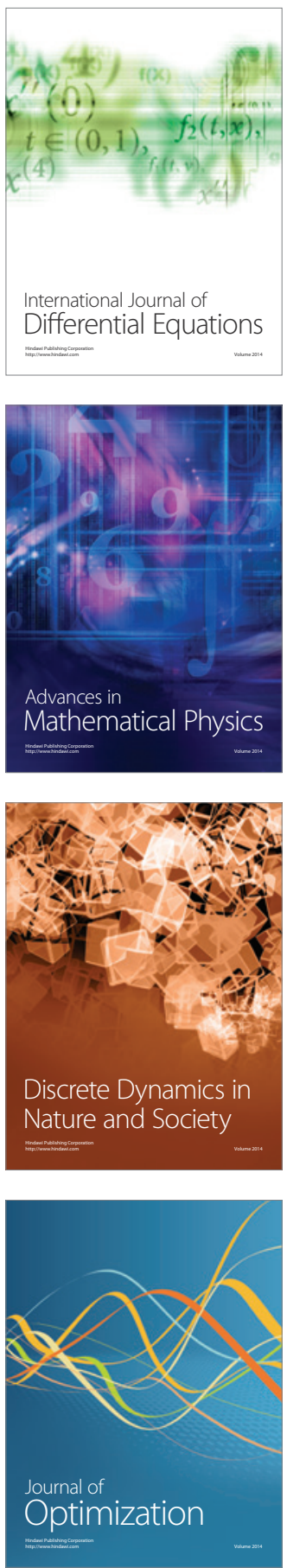